\section{Synthesis of Greatest Linear Feedback for Timed Event Graphs in Dioid}

Bertrand Cottenceau, Laurent Hardouin, Jean-Louis Boimond and Jean-Louis Ferrier

Abstract-This paper deals with the synthesis of greatest linear causal feedback for Discrete Event Systems whose behavior is described in dioid. Such a feedback delays as far as possible the input of the system while keeping the same transfer relation between the input and the output. When a feedback exists in the system, we show how to compute a greater one without decreasing the system's performance.

Keywords - Discrete Event Systems, Dioid, Timed Event Graphs, Feedback Synthesis, Kanban.

\section{INTRODUCTION}

It is well known that the dynamical behavior of Timed Event Graphs (TEG) (a subclass of timed Petri nets which can be used to model deterministic discrete event systems subject to saturation and synchronization phenomena) can be described by a linear model in dioid ([1], [2]). In this context, an element $H \in \mathcal{D}^{m \times n}$ of a matrix dioid can represent the transfer relation between input transitions $U \in \mathcal{D}^{n}$ and output transitions $Y \in \mathcal{D}^{m}$, i.e., $Y=H \otimes U$. This paper deals with the synthesis of a linear feedback $F \in \mathcal{D}^{n \times m}$ such that the process input becomes $V=U \oplus F \otimes Y$ (fig. 1). It has been shown in [1]

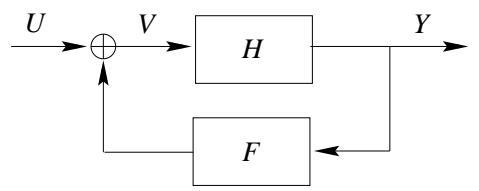

Fig. 1. System with an output feedback F

and [8] that such a feedback allows stabilizing the system (i.e., keeps bounded the number of tokens in the associated TEG). However, this feedback creates new circuits besides the existing ones, therefore the throughput may only decrease. In [1] and [8], it has been stated that any structurally controllable (respectively observable) system, i.e., that any internal transition of the TEG can be reached by a directed path from at least one input transition (respectively is the origin of at least one directed path to some output transition), can be stabilized by a feedback which does not damage the throughput of the system $H$. To ensure this condition, it suffices to place enough tokens in the initial marking of places located on feedback arcs. The minimal number of tokens which allows achieving the objective can be obtained by considering the resource optimization problem which can be reduced to linear programs (as shown in [7]). The purpose of the synthesis given subsequently is slightly different, it is to compute the greatest causal feedback in order to delay as far as possible the input of tokens in the system while keeping the same dynamical behavior $H$; from a manufacturing point of view, this means to reduce the work-in-process by keeping the performance of this production system. In a second step, this principle will be applied to pull flow systems, i.e., systems with a pull control mechanism. In a pull control mechanism, a demand for a finished part of a system activates the

Laboratoire d'Ingénierie des Systèmes Automatisés, 62, avenue Notre-Dame du lac, 49000 ANGERS, FRANCE, Tel: (33) 2413657 33, Fax: (33) 241 3657 35. E-mail: [bertrand.cottenceau, laurent.hardouin, jean-louis.boimond, jean-louis.ferrier]@istia.univ-angers.fr release of a new part into that system. These systems involve clearly a feedback to carry the information from the output to the input. An example of such a system is the Kanban Control System (see [4],[5]). Our purpose is then to replace the existing feedback by the greatest feedback which conserves the same transfer relation as the original system. Most of the results are based on residuation theory and star properties in dioid recalled in section II. The feedback computation is presented in section III and, finally, two examples illustrate the results in section IV.

\section{DioId $\mathcal{M}_{i n}^{a x}[[\gamma, \delta]]$}

Let us recall that a dioid is an idempotent semiring $(\mathcal{D}, \oplus, \otimes)$ (the neutral elements of the operators $\oplus, \otimes$ are denoted respectively $\varepsilon$ and $e$ ). Due to idempotency property, an order relation, denoted $\succeq$, can be associated with $\oplus$ and defined by $a \succeq b \Longleftrightarrow a \oplus b=a$ (i.e., $a \oplus b$ is equal to the least upper bound of $a$ and $b$ ). Such an algebraic structure is very efficient to modelize TEG. The dioid considered in this paper is denoted $\mathcal{M}_{i n}^{a x}[[\gamma, \delta]]$ and is formally the quotient dioid of $\mathbb{B}[[\gamma, \delta]]$, set of formal power series in two variables $(\gamma, \delta)$ with Boolean coefficients and with exponents in $\mathbb{Z}$, by the equivalence relation $x \mathcal{R} y \Longleftrightarrow \gamma^{*}\left(\delta^{-1}\right)^{*} x=\gamma^{*}\left(\delta^{-1}\right)^{*} y$ (with * the Kleene star operator defined as $a^{*}=\bigoplus_{k \geq 0} a^{k}$ ) (see [1],[2] for an exhaustive presentation). It must be noted that equalities are of course not formal equalities but that they express equivalences in the quotient structure $\left(e . g ., e=\gamma^{0} \delta^{0}=\gamma^{*}=\left(\delta^{-1}\right)^{*}=\gamma^{*}\left(\delta^{-1}\right)^{*}\right)$. $\mathcal{M}_{i n}^{a x}[[\gamma, \delta]]$ is complete with a bottom element $\varepsilon=\gamma^{+\infty} \delta^{-\infty}$ and a top element $T=\gamma^{-\infty} \delta^{+\infty}$. Let us consider a representative $s=\bigoplus_{i \in \mathbb{N}} f\left(n_{i}, t_{i}\right) \gamma^{n_{i}} \delta^{t_{i}}$ in $\mathbb{B}[[\gamma, \delta]]$ of an element belonging to $\mathcal{M}_{i n}^{a x}[[\gamma, \delta]]$. The support of $s$ is then defined as $\left\{\left(n_{i}, t_{i}\right) \mid f\left(n_{i}, t_{i}\right) \neq \varepsilon\right\}$ and the valuation (resp. degree) of this element as the lower bound (resp. upper bound) of its support. In $\mathcal{M}_{i n}^{a x}[[\gamma, \delta]]$, owing to its quotient structure, it is meaningless to speak of the degree in $\gamma$ or of the valuation in $\delta$ of one of its elements. Therefore, the valuation (resp. degree) of an element will be its valuation (resp. degree) in $\gamma$ (resp. $\delta$ ).

When an element of $\mathcal{M}_{i n}^{a x}[[\gamma, \delta]]$ is used to code a set of information concerning a transition of a TEG, then a monomial $\gamma^{k} \delta^{t}$ may be interpreted as : the $k^{t h}$ event occurs at least at date $t$. Let us recall that an element $h \in \mathcal{M}_{i n}^{a x}[[\gamma, \delta]]$ is said to be causal either if $(h=\varepsilon)$ or $\left(\operatorname{val}(h) \geq 0\right.$ and $\left.h \succeq \gamma^{v a l(h)}\right)$, i.e., such that it characterizes anticipation neither on time domain nor on the event domain. The algebraic tools used in the next sections are recalled below.

Theorem 1 (see [2]) In a dioid $\mathcal{D}$, the fixed-point equation $x=a x \oplus b$ admits the least solution $x=a^{*} b$.

Proposition 1: Let matrix $A \in \mathcal{D}^{n \times n}$, then

$$
A^{*} A^{m}=A^{m} A^{*} \text {. }
$$

Proof: Due to product associativity (i.e., $A^{k} A^{m}=$ $\left.A^{m} A^{k}\right)$, then $A^{*} A^{m}=A^{m} \oplus A A^{m} \oplus A^{2} A^{m} \oplus \cdots=A^{m}(E \oplus A \oplus$ $\left.A^{2} \cdots\right)=A^{m} A^{*}$ (where $E$ is the identity matrix).

Definition 1 (Residuation) A mapping $f: \mathcal{C} \rightarrow \mathcal{D}$ where $\mathcal{C}, \mathcal{D}$ are ordered sets, is residuated if for all $y \in \mathcal{D}$, the least upper bound of the subset $\{x \in \mathcal{C} \mid f(x) \preceq y\}$ exists and belongs to this subset. It is then denoted $f^{\sharp}(y)$. The mapping $f^{\sharp}: \mathcal{D} \rightarrow \mathcal{C}$ is called the residual of $f$. By definition, $f\left[f^{\sharp}(y)\right] \preceq y, \forall y \in \mathcal{D}$.

Property 1: Let $f: \mathcal{C} \rightarrow \mathcal{D}$ a residuated mapping. If there exists $x$ such that $f(x)=y$, then $f\left(f^{\sharp}(y)\right)=y$.

Remark 1: More generally, with $f$ isotone, if there exists $x$ such that $f(x)=y$ and $\hat{x}$ is the greatest subsolution to $f(x) \preceq y$, then $f(\hat{x})=y$.

Theorem 2 ([2]) In a dioid $\mathcal{D}$, each inequality $a \otimes x \preceq b$ and $x \otimes a \preceq b$ always admit a greatest solution denoted respectively 
$x=a \phi b$ and $x=b \phi a, x \in \mathcal{D}$. Mappings $x \mapsto a \phi x$ and $x \mapsto x \phi a$ are the residuals of $x \mapsto a \otimes x$ and $x \mapsto x \otimes a$.

Theorem 3 ([6]) Let $\mathcal{E}$ an ordered complete set and $\mathcal{F}$ a complete subset of $\mathcal{E}$ with the bottom element of $\mathcal{E}$, denoted $\varepsilon$. Injection $i: \mathcal{F} \rightarrow \mathcal{E}, x \mapsto x$ is residuated. Its residual is denoted $\operatorname{pr}_{\mathcal{F}}=i^{\sharp}$ and satisfies:

$$
\begin{array}{ll}
\text { (i) } & \operatorname{pr}_{\mathcal{F}} \circ \mathrm{pr}_{\mathcal{F}}=\operatorname{pr}_{\mathcal{F}}, \\
\text { (ii) } & \operatorname{pr}_{\mathcal{F}} \preceq I d_{\mathcal{E}}, \\
\text { (iii) } & x \in \mathcal{F} \Longleftrightarrow \operatorname{pr}_{\mathcal{F}}(x)=x .
\end{array}
$$

Application 1: Let $\operatorname{Caus}\left(\mathcal{M}_{i n}^{a x}[[\gamma, \delta]]\right)$ the set of causal elements of $\mathcal{M}_{i n}^{a x}[[\gamma, \delta]]$, namely:

$\operatorname{Caus}\left(\mathcal{M}_{i n}^{a x}[[\gamma, \delta]]\right)=\left\{x \in \mathcal{M}_{i n}^{a x}[[\gamma, \delta]] \mid x=\varepsilon\right.$ or $(\operatorname{val}(x) \geq$ 0 and $\left.\left.\gamma^{\operatorname{val}(x)} \preceq x\right)\right\}$.

Injection $i: \operatorname{Caus}\left(\mathcal{M}_{i n}^{a x}[[\gamma, \delta]]\right) \rightarrow \mathcal{M}_{i n}^{a x}[[\gamma, \delta]], x \mapsto x$ is residuated and its residual is denoted $\mathrm{pr}_{\text {Caus }}$.

Clearly, $\operatorname{pr}_{\text {Caus }}(x)$ is the greatest element of $\operatorname{Caus}\left(\mathcal{M}_{i n}^{a x}[[\gamma, \delta]]\right)$ smaller than or equal to $x \in \mathcal{M}_{i n}^{a x}[[\gamma, \delta]]$ :

$$
\begin{gathered}
\operatorname{pr}_{\text {Caus }}\left(\bigoplus_{i \in \mathbb{N}} f\left(n_{i}, t_{i}\right) \gamma^{n_{i}} \delta^{t_{i}}\right)=\bigoplus_{i \in \mathbb{N}} g\left(n_{i}, t_{i}\right) \gamma^{n_{i}} \delta^{t_{i}} \\
\text { where } g\left(n_{i}, t_{i}\right)=\left\{\begin{array}{l}
f\left(n_{i}, t_{i}\right) \text { if }\left(n_{i}, t_{i}\right) \geq(0,0) \\
\varepsilon \text { otherwise }
\end{array} .\right.
\end{gathered}
$$

Remark 2: the notion of projection in causal set will be simply extended to the matrix dioid case in the next sections. In other words, if $M \in \mathcal{D}^{m \times n}, \operatorname{pr}_{\text {Caus }}(M)$ will be defined as follows

$$
\operatorname{pr}_{\text {Caus }}(M)_{i j}=\operatorname{pr}_{\text {Caus }}\left(M_{i j}\right) i=1, \cdots, m \text { and } j=1, \cdots, n \text {. }
$$

\section{Greatest Linear feEdbaCk Synthesis}

\section{A. Feedback computation}

A system modelized by a TEG and assumed to be structurally controllable and observable may be described by a transfer relation $H$, then

$$
Y=H \otimes U \text {. }
$$

Our purpose is to synthesize the greatest linear feedback $F$ in order to keep the open-loop transfer relation $H$. This feedback allows delaying as far as possible the input of parts, i.e. reducing the work-in-process, by keeping exactly the same production performances. According to fig. 1 and theorem 1, the transfer relation of the closed-loop system is $(H F)^{*} H$ (since $\left.Y=H(U \oplus F Y)=(H F)^{*} H U\right)$. Hence, we first propose to find the greatest feedback $F$ such that $(H F)^{*} H=H \Rightarrow$ $(H F)^{*} H U=H U \forall U$. As said before, by considering the optimization resource problem, a feedback $F$ keeping the open-loop throughput is given in [7]. In the second step, we focus on finding the greatest linear causal feedback denoted $\hat{F}_{c}$, i.e.,

$$
\hat{F}_{c}=\bigoplus\left\{F \in \operatorname{Caus}\left(\mathcal{M}_{i n}^{a x}[[\gamma, \delta]]\right) \mid(H F)^{*} H=H\right\} .
$$

Lemma 1: The greatest solution to $(H x)^{*} H \preceq H$ is given by

$$
\hat{F}=H \nmid H \phi H .
$$

Proof: By considering the Kleene star operator, we obtain the following equivalence

$$
(H x)^{*} H \preceq H \Longleftrightarrow\left\{\begin{array}{l}
H \preceq H \\
H x H \preceq H \\
(H x)^{2} H \preceq H \\
\vdots
\end{array}\right.
$$

Clearly, $H \preceq H$ is satisfied $\forall x$. By applying the residuation theory, the greatest solution of $H x H \preceq H$ is $\hat{F}=H \phi H \phi H$.
Then the solution set $L=\left\{x \mid(H x)^{*} H \preceq H\right\}$ is bounded by $\hat{F}$, i.e., $\forall x \in L, x \preceq \hat{F}$. Furthermore $\hat{F} \in L$. Indeed, $(H \hat{F}) H \preceq H$, then by recalling that the law $\otimes$ is isotone : $(H \hat{F})(H \hat{F}) H \preceq$ $(H \hat{F}) H \preceq H$ and $\forall n,(H \hat{F})^{n} H \preceq(H \hat{F})^{n-1} H \preceq \cdots \preceq H$. Subsequently, $H \oplus \bigoplus_{n \in \mathbb{N}^{*}}(H \hat{F})^{n} H=(H \hat{F})^{*} H \preceq H, \hat{F}$ is then the greatest element of $L$.

Corollary 1: $\hat{F}=H \phi H \phi H$ is the greatest solution to the equation $(H x)^{*} H=H$.

Proof: The matrix $\varepsilon$ is solution to this equation. Therefore, according to property 1 and remark $1, \hat{F}$ is solution too and the greatest one.

Proposition 2: The greatest linear and causal feedback allowing keeping the open-loop behavior is given by :

$$
\hat{F}_{c}=\operatorname{pr}_{\text {Caus }}(\hat{F})=\bigoplus\left\{x \in \operatorname{Caus}\left(\mathcal{M}_{i n}^{a x}[[\gamma, \delta]]\right) \mid(H x)^{*} H=H\right\} .
$$

Proof: From theorem 3 (ii) and its application, we have $\hat{F}_{c}=\operatorname{pr}_{\text {Caus }}(H \phi H \phi H) \preceq \hat{F}$. Therefore, $\left(H \hat{F}_{c}\right)^{*} H \preceq(H \hat{F})^{*} H \preceq$ $H$. However, $\left(H \hat{F}_{c}\right)^{*} \succeq E$ because of Kleene star definition, eventually $H \preceq\left(H \hat{F}_{c}\right)^{*} H \preceq(H \hat{F})^{*} H \preceq H$, then $\left(H \hat{F}_{c}\right)^{*} H=$ $H$. It is clear from theorem 3 that it cannot exist a causal element greater than $\hat{F}_{c}$ and smaller than $\hat{F}$.

Remark 3: Let us note that it would be an interesting extension of this work to address the problem of feedback synthesis in order to obtain a closed-loop behavior, say $G$ (reference model), different from the open-loop one, namely $H$ (obviously such that $G \succeq H)$.

\section{B. Pull flow systems}

In this section, we will focus on particular systems with pull flow control. In these systems, an existing causal feedback $F$ allows releasing firing of input transitions, which can be represented by the block-diagram shown in fig. 2 where $Y_{c}$ represents the customer's demand and $U$ the stock of unprocessed parts. Our purpose is to replace $F$ by the greatest causal feedback $\hat{F}_{p_{c}} \succeq F$ which keeps the same dynamical behavior and delays as far as possible the input of unprocessed parts. The transfer

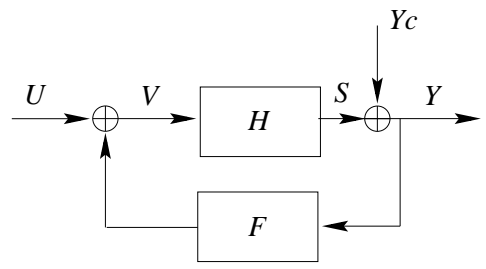

Fig. 2. System with pull flow control mechanism

relation of such a system is

$$
Y=(H F)^{*} Y_{c} \oplus(H F)^{*} H U
$$

$S$ represents the dynamical behavior of the finished parts (i.e., the dates at which the finished parts are put in the downstream stock ). Its behavior is described by

$$
S=(H F)^{*} H F Y_{c} \oplus(H F)^{*} H U
$$

Classically, $F$ is computed in order to minimize a cost function depending on the holding and backordering inventory. In a pull flow system, $S$ is more representative of the system behavior than $Y$. Indeed, there is a marge between customer's demand and finished parts that we must conserve. Therefore, our purpose is to find a causal feedback $\hat{F}_{p_{c}}$ greater than or equal to $F$ allowing keeping the same behavior for $S$ in regards to $Y_{c}$. 
This means to reduce as far as possible the work-in-process by keeping the same stock of finished part, then by satisfying the same customer's demand. Formally, we will search the greatest $\hat{F}_{p_{c}}$ such that $\left(H \hat{F}_{p_{c}}\right)^{*} H \hat{F}_{p_{c}}=(H F)^{*} H F$.

Remark 4: the same work may be done by taking account of output behavior instead of $S$ behavior.

Lemma 2: The greatest solution to $(H x)^{*} H x \preceq(H F)^{*} H F$ is given by

$$
\hat{F}_{p}=H \nmid\left((H F)^{*} H F\right) .
$$
we have

Proof: By considering the same arguments as in lemma 1,

$$
(H x)^{*} H x \preceq(H F)^{*} H F \Longleftrightarrow\left\{\begin{array}{l}
H x \preceq(H F)^{*} H F \\
(H x)^{2} \preceq(H F)^{*} H F \\
\vdots
\end{array}\right.
$$

The greatest solution of $H x \preceq(H F)^{*} H F$ is given using residuation theory by $\hat{F}_{p}=H \phi\left((H F)^{*} H F\right)$. We deduce that the solution set $L=\left\{x \mid(H x)^{*} H x \preceq(H F)^{*} H F\right\}$ is bounded by $\hat{F}_{p}$. Furthermore, we show that $\hat{F}_{p} \in L$. Indeed, assuming that $\left(H \hat{F}_{p}\right)^{n} \preceq(H F)^{*}(H F)^{n}$,

$$
\begin{aligned}
\left(H \hat{F}_{p}\right)^{n+1} \preceq & (H F)^{*}(H F)^{n} H\left(H \phi\left((H F)^{*} H F\right)\right) \\
\left(H \hat{F}_{p}\right)^{n+1} \preceq & (H F)^{*}(H F)^{n}(H F)^{*} H F \\
& \quad\left(\text { since } A^{*} A^{m}=A^{m} A^{*}\right. \text { (proposition 1) } \\
& \quad \text { and } A(A \oint(A X))=A X) \\
\left(H \hat{F}_{p}\right)^{n+1} \preceq & (H F)^{*}(H F)^{n+1}
\end{aligned}
$$

(by applying proposition 1 and $A^{*} A^{*}=A^{*}$ )

Moreover, for $n=0$, we have $H \hat{F}_{p} \preceq(H F)^{*}(H F)$, then $\forall i \geq 1$, $\left(H \hat{F}_{p}\right)^{i} \preceq(H F)^{*}(H F)^{i}$. By summing these inequalities for all $i$, we have $\bigoplus_{i \geq 1}\left(H \hat{F}_{p}\right)^{i} \preceq \bigoplus_{i \geq 1}(H F)^{*}(H F)^{i}$, that can be rewritten $\left(H \hat{F}_{p}\right)^{*} H \hat{F}_{p} \preceq(H F)^{*} H F$, i.e., $\hat{F}_{p} \in L$, hence it is the greatest solution of (3) since it belongs to $L$ and it is its upper bound.

Corollary 2: $\hat{F}_{p}=H \phi\left((H F)^{*} H F\right)$ is the greatest solution to

$$
(H x)^{*} H x=(H F)^{*} H F \text {. }
$$

Proof: It suffices to remark that $F$ is a solution to equation (4). Therefore, according to property 1 and remark $1, \hat{F}_{p}$ is the greatest solution to (4).

Proposition 3: The greatest linear and causal feedback allowing keeping the behavior of $S$ in the pull control system is given by

$$
\hat{F}_{p_{c}}=\operatorname{pr}_{\text {Caus }}\left(\hat{F}_{p}\right) \text {. }
$$

Proof: We will show here that this greatest causal feedback conserves the same transfer relation as the one obtained with $F$, i.e. $S=(H F)^{*} H F Y_{c} \oplus(H F)^{*} H U=\left(H \hat{F}_{p_{c}}\right)^{*} H \hat{F}_{p_{c}} Y_{c} \oplus$ $\left(H \hat{F}_{p_{c}}\right)^{*} H U$. Hence, we will proceed in two steps.

Fisrtly, from corollary $2, \hat{F}_{p} \succeq F$. Since $\operatorname{pr}_{\text {Caus }}$ is residual mapping, it is isotone. Then $\operatorname{pr}_{\text {Caus }}\left(\hat{F}_{p}\right) \succeq \operatorname{pr}_{\text {Caus }}(F)$. Moreover, $F$ is assumed causal, then according to theorem $3, \operatorname{pr}_{\text {Caus }}(F)=$ $F$. From theorem 3 , we deduce that $\operatorname{pr}_{\text {Caus }}\left(\hat{F}_{p}\right) \preceq \hat{F}_{p}$. Then, $\operatorname{pr}_{\text {Caus }}\left(\hat{F}_{p}\right)$ is bounded :

$$
F \preceq \operatorname{pr}_{\text {Caus }}\left(\hat{F}_{p}\right) \preceq \hat{F}_{p} \quad\left(\text { i.e., } F \preceq \hat{F}_{p_{c}} \preceq \hat{F}_{p}\right) .
$$

Therefore, by using isotony of mapping $(H x)^{*} H x$, we deduce $(H F)^{*} H F \preceq\left(H \hat{F}_{p_{c}}\right)^{*} H \hat{F}_{p_{c}} \preceq\left(H \hat{F}_{p}\right)^{*} H \hat{F}_{p}=(H F)^{*} H F$ (see corol. 2).

In other words, this feedback keeps the transfer relation between $Y_{c}$ and $S$.
On the other hand, $\hat{F}_{p}$ is such that $\left(H \hat{F}_{p}\right)^{*} H=$ $\left((H F)^{*} H F\right)^{*} H=(H F)^{*} H$ (i.e., the greatest feedback $\hat{F}_{p}$ keeps the transfer relation between $U$ and $S$ ). Since $\hat{F}_{p} \succeq F$ and $\operatorname{pr}_{\text {Caus }}(F)=F$, it may be shown similarly

$$
\left(H \operatorname{pr}_{\text {Caus }}\left(\hat{F}_{p}\right)\right)^{*} H=(H F)^{*} H
$$

i.e., the transfer relation between $U$ and $S$ is also kept with feedback $\hat{F}_{p_{c}}$.

Remark 5: keeping the behavior of $S$ implies keeping the behavior of $Y$ too.

\section{Illustrative EXAMPLES}

Example 1: In a first step, we will apply the results concerning the greatest feedback computation (results presented in III-A) by considering a similar example as the one presented in [1] and [8]. The TEG considered is drawn in fig. 3 in solid lines. Its transfer matrix is $H=\left[\delta^{5}\left(\gamma^{2} \delta^{3}\right)^{*} \delta^{5}\left(\gamma^{2} \delta^{3}\right)^{*}\right]$. According

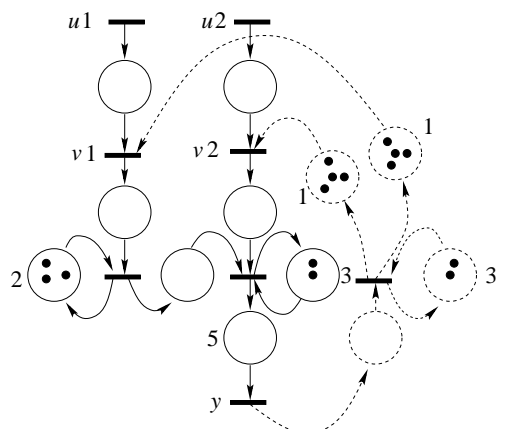

Fig. 3. MIMO TEG

to corollary 1 , the greatest feedback keeping the same transfer relation is given by

$$
\hat{F}=H ф H \phi H=\left[\delta^{-5}\left(\gamma^{2} \delta^{3}\right)^{*} \delta^{-5}\left(\gamma^{2} \delta^{3}\right)^{*}\right]^{T} .
$$

Clearly, this feedback is not causal. So, the greatest causal feedback which keeps the same output behavior as the openloop system (according to proposition 2) is

$$
\hat{F}_{c}=\operatorname{pr}_{\text {Caus }}(\hat{F})=\left[\gamma^{4} \delta^{1}\left(\gamma^{2} \delta^{3}\right)^{*} \gamma^{4} \delta^{1}\left(\gamma^{2} \delta^{3}\right)^{*}\right]^{T} .
$$

Remark 6: Let us note that the initial system becomes stable with the greatest feedback $\hat{F}_{c}$ (in doted lines in fig. 3). According to the results given in [1] and [8], it is known that a feedback "increases" stability since it creates new circuits. An interesting question is to determine the conditions on system $H$ such that feedback $\hat{F}_{c}$ stabilizes the system.

Remark 7: Considering the example proposed in [1] and [8] in which only values differ, we obtain $\hat{F}_{c}=\left[\gamma(\gamma \delta)^{*} \gamma(\gamma \delta)^{*}\right]^{T}$ which is to compare with $F=[\gamma \gamma]^{T}$ obtained by the authors. It must be noted that the main difference between these two solutions is the dynamic on $\hat{F}_{c}$.

Example 2: The results about the greatest causal feedback synthesis for a pull flow system are now applied to the well known kanban cell (see [3], [6] where a model and a study of such a system have been given).

Under the assumption that $K \geq n$, i.e. there are more kanbans $(K)$ than machines $(n)$ in the cell, the transfer relation of the kanban cell is given by

$$
y=\left(e \oplus \gamma^{K} \delta^{t}\left(\gamma^{n} \delta^{t}\right)^{*}\right) y_{c} \oplus \delta^{t}\left(\gamma^{n} \delta^{t}\right)^{*} u, \text { (see [6] appendix A) }
$$


i.e., $h=\delta^{t}\left(\gamma^{n} \delta^{t}\right)^{*}$ and $f=\gamma^{K}$. According to corollary 2, the optimal feedback which keeps the behavior unchanged is $\hat{f}_{p}=$ $h \phi\left((h f)^{*} h f\right)=\left(\delta^{t}\left(\gamma^{n} \delta^{t}\right)^{*}\right) \phi\left(\left(\gamma^{K} \delta^{t}\left(\gamma^{n} \delta^{t}\right)^{*}\right)^{*} \gamma^{K} \delta^{t}\left(\gamma^{n} \delta^{t}\right)^{*}\right)$. Since $\left(a b^{*}\right)^{*} a b^{*}=a(a \oplus b)^{*}$ (see [6] (4.1.6)), then $\hat{f}_{p}=$ $\left(\delta^{t}\left(\gamma^{n} \delta^{t}\right)^{*}\right) \phi\left(\gamma^{K} \delta^{t}\left(\gamma^{K} \delta^{t} \oplus \gamma^{n} \delta^{t}\right)^{*}\right)$. Therefore, under the assumption $K \geq n, \hat{f}_{p}=\gamma^{K}\left(\gamma^{n} \delta^{t}\right)^{*}$.

Clearly, this feedback is causal. Indeed, the greatest causal feedback less than or equal to $\hat{f}_{p}$ is

$$
\hat{f}_{p_{c}}=\operatorname{pr}_{\text {Caus }}\left(\hat{f}_{p}\right)=\gamma^{K}\left(\gamma^{n} \delta^{t}\right)^{*}=\hat{f}_{p} .
$$

This new feedback is represented in doted lines on fig. 4 .

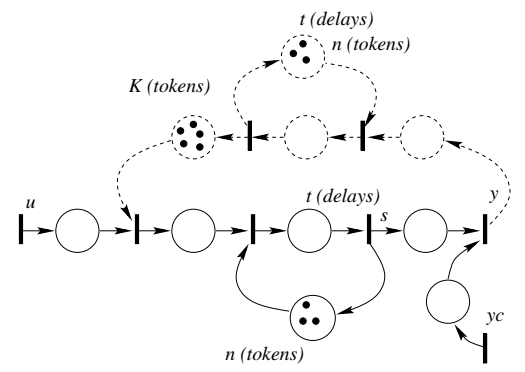

Fig. 4. Kanban cell of the feedback has been modified

\section{Conclusion}

First, we have presented a method to synthesize the greatest linear and causal feedback in order to keep the transfer relation of the open-loop system. Secondly, we have proposed a method to modify a pull control system in order to delay as far as possible the input of unprocessed parts without changing the output in regard to the customer's demand. Both methods allow reducing the work-in-process without changing the system performance. They are based on residuation theory and dioid properties. The solutions proposed to solve the two previous problems are relatively reminiscent with the pole placement method well known in the conventional linear system theory.

\section{ACKNOWLEDGMENTS}

The authors would like to thank the referees for their helpful suggestions and comments. They are grateful to C. Ferrier too for her valuable linguistic help.

\section{REFERENCES}

[1] F. Baccelli, G. Cohen, G.J. Olsder, and J.P. Quadrat. Synchronization and Linearity: An Algebra for Discrete Event Systems. Wiley and Sons, 1992.

[2] G. Cohen, P. Moller, J.P. Quadrat, and M. Viot. Algebraic Tools for the Performance Evaluation of Discrete Event Systems. IEEE Proceedings: Special issue on Discrete Event Systems, 77(1):39-58, January 1989.

[3] B. Cottenceau, L. Hardouin, and J.-L. Boimond. Dynamic Control of a Kanban System in Dioid Algebra. Proc. of 5th IEEE Medit. Conf. on Cont. and Syst, Paphos, Cyprus, July 1997.

[4] Y. Dallery, Z. Liu, and D. Towsley. Properties of Fork/Join Queueing Networks with Blocking Under Various Operating Mechanisms. IEEE Trans. Robot. Automat., 13(4):503-518, August 1997.

[5] M. Di Mascolo, Y. Frein, and Y. Dallery. An Analytical Method for Performance Evaluation of Kanban Controlled Production Systems. Operations Research, 44(1):50-64, January-February 1996.

[6] S. Gaubert. Théorie des Systèmes Linéaires dans les Dioüdes. Thèse, École des Mines de Paris, July 1992.

[7] S. Gaubert. Resource Optimization and (min,+) Spectral Theory. IEEE Trans. on Automatic Control, 40(11), November 1995.

[8] M. Plus. Second Order Theory of Min-linear Systems and its Application to Discrete Event Systems. In Proceedings of the 30th $C D C$, Brighton, England, December 1991. 